\title{
Archéopages
}

Archéopages

Archéologie et société

40 | 04-07/2014

Villages

\section{La question des « villages » en Gaule romaine. Entre débat sur les mots et données archéologiques}

\section{Martial Monteil}

\section{(2) OpenEdition}

12 Journals

Édition électronique

URL : https://journals.openedition.org/archeopages/597

DOI : 10.4000/archeopages. 597

ISSN : 2269-9872

\section{Éditeur}

INRAP - Institut national de recherches archéologiques préventives

\section{Édition imprimée}

Date de publication : 1 juin 2015

Pagination : 50-55

ISSN : $1622-8545$

\section{Référence électronique}

Martial Monteil, «La question des « villages » en Gaule romaine. Entre débat sur les mots et données archéologiques », Archéopages [En ligne], 40 | 04-07/2014, mis en ligne le 01 juillet 2016, consulté le 22 janvier 2022. URL : http://journals.openedition.org/archeopages/597 ; DOI : https://doi.org/10.4000/ archeopages.597 


\section{La question des « villages " en Gaule romaine Entre débat sur les mots et données archéologiques}

Martial Monteil Universitéde Nantes, LARA-UMR 6560 «Centre de recherche en archéologie, archéosciences, histoire»

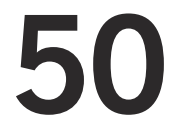

Une façon d'encadrer le propos qui suit, en forçant un peu le trait, est de noter que les archéologues qui s'intéressent à la fin de la Protohistoire et au haut Moyen Âge ont en commun de classer les agglomérations selon deux grandes catégories : les villages d'une part et les villes - ou oppida - d'autre part. Pour la Gaule préromaine, exception faite de sa partie méridionale (Garcia, 2004; Py, 2012), les villages dits «ouverts» - parfois encore qualifiés de vici par référence au De Bello Gallico de César correspondent ainsi à des habitats groupés caractérisés par une fonction dominante, agricole ou artisanale. Mais, en réalité, les artisans paraissent y être plus présents que les paysans (Fichtl, 2005, 2013). Pour le premier Moyen Âge, une fois le poncif du village né au XI ${ }^{\mathrm{e}}$ siècle évacué, une certaine unanimité s'est fait jour pour désigner ainsi des agglomérations dont la fonction principale est agropastorale, éventuellement complétée par des activités artisanales ponctuelles ou en lien avec l'extraction de matières premières (Schneider, 2007 ; É. Peytremann dans ce volume). Pour autant, les uns et les autres ne négligent pas la complexité et la diversité des formes de ce type d'habitat et explorent de nouvelles voies, notamment celle de l'analyse spatiale.

\section{Le poids des mots}

Au contraire, chez les antiquisants, la terminologie appliquée aux nombreuses agglomérations qui maillent les cités des Gaules romaines, hors villes-capitales, a fait - et fait encore - l'objet de multiples débats. Les discussions d'ordre sémantique associées à la recherche sur l'habitat groupé constituent ainsi un épais dossier, que d'aucuns pourront trouver abscons ou très «franco-français», mais qu’on ne peut faire l'économie d'entrouvrir ici, avec évidemment les quelques raccourcis qu'impose le calibrage de cette contribution.

Cela tient en partie à la diversité de ces agglomérations - en termes de morphologie, d'activités identifiées, de parure monumentale attestée ou non, etc. - à la connaissance superficielle d'un grand nombre d'entre elles ou encore à l'impossibilité de leur assigner une terminologie latine acceptée par tous. Il est vrai que les mots latins utilisables dans le domaine de «l'urbain»- et donc contemporains des sites identifiés par l'archéologie - sont somme toute peu nombreux - moins d'une dizaine -, empreints, pour les historiens et les épigraphistes, d'une forte valeur institutionnelle et soumis à des évolutions de sens entre le début et la fin de l'Antiquité (Leveau, 1993, p. 279-282; Tarpin, 1999, 2002, 2009; Hervé, 2012). On ne sait d'ailleurs pas - et on ne saura jamais - quelle signification ces mots - oppidum, forum, castellum, municipium, vicus... - pouvaient avoir pour les populations des provinces gauloises ni même si l'idée d'établir une distinction entre des agglomérations d'ampleur différente leur était familière.

Ainsi, lorsque l'enquête sur les agglomérations des Gaules et des Germanies s'est véritablement développée, à la suite d'un colloque précurseur organisé à l'université de Tours en 1975, une première étape a consisté à privilégier le vocable latin «vicus» (Chevallier, 1976). On lui a ensuite préféré le terme d' «agglomération secondaire», apparu sous la plume de Christian Goudineau en 1980 (Février et al., 1980, p. 101, 104), puis repris avec la première étude consacrée à l'ensemble du réseau urbain antique d'une région, 
la Franche-Comté (Mangin et al., 1986, p.18). Ce «vocabulaire d'attente», considéré comme approprié pour recouvrir la diversité des formes des agglomérations et adapté à une documentation souvent lacunaire, a été rapidement admis et réutilisé, notamment à l'occasion d'une synthèse régionale portant sur l'Aquitaine (Mangin, Tassaux, 1992), puis du colloque de Bliesbruck-Reinheim/ Bitche en octobre 1992 (Petit, Mangin, 1994). Il a été conservé dans la plupart des synthèses ultérieures, en retenant la définition suivante : « tout site archéologiquement attesté qui se situe entre la ferme ou la villa isolée et la capitale de cité, c'est-à-dire du village de paysans et de la station routière modeste à l'agglomération dont le paysage est très proche de celui de la ville chef-lieu de cité» (Petit et al., 1986, p.18). En bref, il s'agit d'une caractérisation par la négative - les villes-capitales ainsi que les formes de l'habitat dispersé sont exclues du propos - et qui rassemble des sites distribués suivant un gradient qui va du plus ou moins rural au plus ou moins urbain. Enfin, pour mieux organiser la documentation, une typologie a été rapidement mise en œuvre, permettant de classer ces agglomérations selon plusieurs types, dont l'un réunissant «l'agglomération rurale ou village » (voir, par exemple, Mangin, Tassaux, 1992, p. 466).

Récemment, le débat a repris à deux niveaux, le premier sur l'usage des mots et les idées qu'ils sous-tendent, le second sur la nécessité d'emprunter de nouvelles voies. Quatre contributions publiées dans les actes de la table ronde d'Orléans, consacrée en 2004 aux «agglomérations secondaires antiques en région Centre», résument fort opportunément les grandes lignes de la discussion et fournissent la bibliographie antérieure. Philippe Leveau y revient ainsi sur sa proposition de remplacer le vocable «agglomération secondaire» par le mot latin «vicus», dont la pluralité des sens - notamment celui de «bourg» ou de «village» - en fait un synonyme du précédent et dont il estime qu'il ne relève pas exclusivement de la terminologie administrative (Leveau, 2012) ${ }^{\mathbf{1}}$. Michel Tarpin rappelle cependant que «vicus» relève du vocabulaire institutionnel et non d'une réalité matérielle, et qu'il ne peut donc être appliqué de manière systématique à toutes les agglomérations (Tarpin, 2012). Monique Dondin-Payre, quant à elle, réfute toute équivalence entre «vicus » et «agglomération secondaire» et suggère d'employer désormais les seuls termes de «localité » ou « d'agglomération », sans adjectif associé (DondinPayre, 2012). Enfin, Pierre Garmy revient sur l'impasse que constitue à son sens la typologie, surtout en utilisant un vocabulaire contemporain, et sur la nécessité, pour en sortir, de continuer à développer les outils et concepts empruntés aux géographes, notamment la modélisation et l'approche systémique (Garmy, 2012a; voir aussi Garmy, 2012b) ${ }^{2}$.

Il faut noter que les prises de position qui viennent d'être brièvement résumées intègrent toutes la question du «village», de manière différente cependant et parfois avec réticence en raison de l'étiquette médiévale qui lui est accolée, bien qu'il n'y ait aucune raison que les médiévistes aient un droit réservé sur ce terme. Les catalogues raisonnés de sites et les synthèses fondées sur la typologie ont ainsi, pour la plupart, pris en compte le village et sa fonction primaire, agricole ou liée à l'extraction de matières premières (par exemple Mangin et al., 1986, p. 219, 226-228; Massy, 1997, p.421-422). Il en a été de même dans le cadre de la démarche chronotypologique mise en œuvre en Languedoc-Roussillon (Raynaud, 2002, p. 41; 2003, p. 331). Par ailleurs, le mot «vicus» aurait, parmi d'autres traductions révélatrices de sa pluralité, celle de village, mais le fait n'est pas unanimement admis (Leveau, 2012, p. 167; contra Tarpin, 2012, p. 178). Enfin, la hiérarchisation des réseaux urbains prend aussi en compte les formes agglomérées les plus modestes, situées aux franges de «l'infra-urbain» (Garmy, 2012a, p. 184).

\section{Du sens des mots à la réalité archéologique}

On peut considérer le débat sur le village au sein des Gaules romaines comme dépourvue de signification, si on admet que toute tentative de typologie est promise à l'échec ou se traduit par « un fatras inextricable, et surtout inopérant» de termes réducteurs (Garmy, 2012b, p. 20). Il doit pourtant être possible de suivre une voie médiane entre le «tout typologie» et le «tout spatial», l'intérêt de cette dernière méthode étant d'ailleurs indiscutable. On peut en effet considérer que « la typologie reste un outil indispensable à la réflexion, ne serait-ce que pour faire apparaître la complexité des situations » (Leveau, 2005-2006, p.159). Plus récemment, François Favory a préconisé la même approche et suggéré de revenir au vocabulaire de la géographie contemporaine - hameau, village, bourg - pour désigner les agglomérations les plus «rurales », parce qu'il lui paraît adapté à l'état actuel des connaissances, susceptible de faire progresser l'enquête et qu'il présente aussi l'avantage d'être mieux compris de tous (Favory, 2012). Il a été entendu par Philippe Leveau, ce dernier notant toutefois que «ces types sont des entités abstraites sur les caractéristiques desquelles il convient de s'accorder. Car chacun de ces termes a un sens très relatif aussi bien dans l'espace que dans le temps et aucun n'est universel » (Leveau, 2013, p.10).

Tentons donc l'exercice, en reprenant le type «village» et en mesurant évidemment les risques à en définir le contenu antique sur la base d'un mot d'origine médiévale et d'une classification moderne et contemporaine. On admettra comme postulat de base qu'un village est un habitat groupé dont l'essentiel de la population a pour activité principale l'agriculture et/ou l'élevage, ce qui n'exclut pas quelques autres occupations plus ponctuelles ${ }^{3}$. Un parfait synonyme serait donc celui
1. Le mot «villa», qui,
rappelons-le, a donné

logiquement situés à

Iinterface entre habitat

parfois qualifiés d'habitats

«intercalaires» et donc

sujets a discussion (vo

2003, p. 331; Raynaud et

al., 2009, p. 158). 
« d'agglomération rurale» (Vidal de la Blache, 1922, p.180-182; Brunet et al., 1993, p. 21, 484), vocable cependant généralisé chez les archéologues antiquisants sans forcément désigner un village ${ }^{4}$. $\mathrm{Si}$ on poursuit sur ce registre, un village devrait supposer non seulement un regroupement d'unités d'habitations, mais aussi, comme dans les villae et les fermes, des témoignages avérés d'activités agropastorales: outils agricoles en nombre, espaces dédiés au stockage des récoltes ou à la stabulation, équipements destinés à la transformation de la production, etc.

Ce principe est opérant si, bien sûr, le village n'est pas seulement un réservoir de main-d'œuvre pour les domaines détenus par les élites. À ce titre, le lien de proximité entretenu entre des agglomérations et une ou plusieurs villae, déjà souligné dès les premières enquêtes en se fondant sur l'exemple de Bliesbruck-Reinheim (Moselle et Sarre), a été mis en relation avec une phrase attribuée à Frontin (De controversiis agrorum, p.53) - ou au pseudo Agennius Urbicus. Elle décrit une situation en Afrique du Nord où «les propriétaires privés possèdent dans ces domaines une population plébéienne nombreuse ainsi que des vici entourant les villae comme un rempart». Il y aurait là un processus expliquant la difficulté à distinguer villa et vicus et permettant de définir «l'agglomération rurale ou villageoise» (Leveau, 2002, p. 23 ; 20052006, p. 160). Certes, ce genre de configuration, avec association entre une agglomération et une ou plusieurs villae, est de plus en plus fréquemment observé en Narbonnaise (Leveau, Garmy, 2002), dans la cité de Vienne (Leveau, Rémy, 2005-2006) ou dans les Trois Gaules (Ferdière, 1999, p.19; Ferdière et al., 2010, p. 403-404).

Pour autant, la chronologie est rarement assez précise pour affirmer que les villae sont antérieures aux habitats groupés. Bliesbruck-Reinheim, qui n'a pas grand-chose à voir avec une agglomération peuplée de paysans, en constitue d'ailleurs un contre-exemple, puisque les quartiers artisanaux sont occupés dès les années 40-50 de notre ère, alors que la villa n'est établie qu'à la fin du ${ }^{\mathrm{er}}$ siècle (Petit, 2005, p. 88, 172). Pour aller plus loin, disons sans doute qu'il est raisonnable d'imaginer que les paysans pouvaient être logés au sein des villae, dans les fermes avoisinantes et dans certaines agglomérations, mais sans que ces dernières soient pour autant dépendantes et exclusivement conçues comme des regroupements de travailleurs agricoles (voir, dans le même sens, Garmy, 2002, p.30; Ferdière et al., 2010, p. 404).

À ce stade, il faut rappeler que bon nombre de spécialistes du monde rural gallo-romain considèrent que l'essentiel de l'économie agraire reposait en fait sur des établissements dispersés dans les campagnes, fermes et villae, tout en notant, sans autre précision, que des habitats groupés pouvaient également jouer un rôle dans ce domaine (Ouzoulias, 2010, p.196; Fiches et al., 2013, p. 371). Mais un récent bilan souligne que «la question de l'existence de véritables villages ruraux, à fonctions agropastorales, dans les campagnes de la Gaule, fait toujours débat» et n'énumère que quelques rares cas avérés (Ferdière, 2013; voir aussi Ferdière, 2005a, p. 239; infra). Parmi ceux-ci, quelques-uns prêtent d'ailleurs à discussion.

Le plus emblématique est celui de Lunel-Viel (Hérault), dont la fouille a donné lieu à la première monographie portant en titre la mention de «village gallo-romain » (Raynaud, 1990) ${ }^{\mathbf{5}}$.

Les quartiers fouillés, qui représentent environ un tiers d'un site d'à peine plus de 1 ha, réunissent à leur apogée, à la fin du $\mathrm{I}^{\mathrm{er}}$ siècle de notre ère, des rues formant carrefour, deux thermes publics, dont l'un avec palestre, un autre édifice public connu seulement par un chapiteau corinthien, plusieurs habitations, dont certaines à pièces multiples et cour centrale (domus), ainsi que des zones cultivées en périphérie sous la forme de jardins et de champs. S'y ajoutent des inscriptions trahissant la présence en ces lieux de notables exerçant leur magistrature à Nîmes, tandis que la part des installations dédiées au stockage ou à l'élevage reste congrue et sujette à hypothèse et que l'importance des activités agricoles demeure difficile à mesurer, même si les études de la faune et de l'outillage l'accréditent. Tout est affaire d'interprétation, mais un tel site, aussi modeste en superficie soit-il, est-il un vrai village, alors qu'il passerait aisément comme une agglomération ayant des traits urbains affirmés dans d'autres territoires des Gaules romaines? Il faut en fait rappeler que Claude Raynaud a utilisé ce terme après avoir exclu par prudence celui de vicus, préférant l'appellation plus neutre «d'habitat groupé, d'agglomération ou plus proche d'une réalité médiévale et actuelle, celle de village», cette dernière l'emportant (Raynaud, 1990, p. 281). Quelques années plus tard, il indiquait qu'évidemment Lunel-Viel pouvait être intégré dans des catégories différentes, suivant qu'on mettait en avant sa position de carrefour routier et ses établissements publics ou, à l'inverse, sa faible superficie et le caractère modeste de quelques-unes de ses maisons (Raynaud, 2002, p. 41). Enfin, plus récemment encore, il a expliqué « la nécessité de la notion de "village gallo-romain" pour désigner une forme, l'une des formes les plus modestes de regroupement antique, émergeant à peine de l'habitat rural» (Raynaud, 2007, p. 292).

Dans cette dernière acception, bon nombre d'agglomérations antiques pourraient donc être qualifiées de villages, mais l'exemple de Lunel-Viel ne correspond pas pleinement au postulat établi plus haut ${ }^{6}$.

Toujours dans le midi de la France, la mise en évidence, sur la frange orientale de l'agglomération de Peyre-Plantade (Clermont-l'Hérault, Hérault), de plusieurs unités de production vinicole au contact de vignobles (Pomarèdes et al., 2008, p.14-24), tout comme à Berthoire (Pignans, Var) (Brun, 
Borréani, 1999, p.556-560), atteste la présence d'une communauté de viticulteurs. Mais ces deux sites livrent des témoignages d'autres activités et des vestiges d'autres types de bâtiments qui, à mon sens, empêchent de les qualifier de villages. Il y aurait là, plutôt, des quartiers périphériques placés directement au contact des terres agricoles et dont la spécialité - ici à forte valeur ajoutée ajoute encore à la diversité des fonctions mises en évidence au sein des agglomérations.

\section{Et le village gallo-romain dans tout ça?}

Quelques exemples extraits d'inventaires et synthèses récents paraissent en réalité confirmer la rareté des villages, dans l'acception étroite du mot. Ainsi, cette forme agglomérée reste à identifier en Aquitaine (Mangin, Tassaux, 1992, p. 465 ; Bouet, Tassaux, 2005, p. 267), tout comme en Bretagne et Pays de la Loire (Monteil, 2012), dans la région Centre (Cribellier, 2013) ou encore dans le Massif central (Baret, 2013). Elle est peu assurée, à quelques exceptions près, en Lorraine (Massy, 1997, p. 421) et en Languedoc-Roussillon (Fiches, 2002), y compris dans la cité de Nîmes où on compte trois «centres ruraux», en incluant LunelViel, pour une soixantaine d'agglomérations recensées (Bermond et al., 2013, p. 84-85). À l'inverse, la grande majorité des agglomérations bénéficiant d'un niveau de connaissance élevé révèlent peu d'activités directement en lien avec l'exploitation de la terre. Un seul exemple peut illustrer ce constat, celui de Jouars-Pontchartrain (Yvelines), le seul vicus sûrement attesté par l'épigraphie dans la cité des Carnutes. Il s'étend sur 30 à 40 ha, est doté d'un sanctuaire assez vaste et a livré des témoignages multiples de pratiques artisanales, d'activités de boucherie et de charcuterie, de commerces - dont de probables horrea -, tandis que les installations à vocation agropastorale restent à trouver (Blin, 2007). Il convient cependant de nuancer le propos, car les vestiges d'activités agropastorales peuvent être plus difficiles à percevoir, lorsqu'ils ne se présentent pas sous une forme clairement structurée, que ceux qui sont en relation avec l'artisanat ou que les éléments relevant de l'urbain.

À ce stade de l'enquête, l'inventaire des villages « de paysans » qui peut être proposé n'est guère plus étoffé que ceux qui l'ont précédé et renvoie, pour l'essentiel, à des zones situées aux confins des provinces gauloises et germaniques ou à des espaces de moyenne montagne (Ferdière, 2005a, p. 239; 2013; Van Ossel, 2010, p. 232).

Il en est ainsi, à l'interface entre Gaule Belgique et Germanie supérieure, sur le versant nord-ouest des Vosges et autour du col de Saverne, de villages et hameaux regroupant des fermes et associés à des parcellaires fossiles. Les activités agropastorales semblent dominantes en leur sein avec, suivant les secteurs, une exploitation ponctuelle de carrières de grès, voire de forêts (Pétry, 1997). Les villages les mieux connus sont ceux du Wasserwald
(Haegen, Moselle) et de la Croix-Guillaume (SaintQuirin, Moselle), mais de multiples nuances ont cependant été introduites récemment sur ces sites longtemps associés à une «culture des sommets vosgiens », sans cependant remettre en question leur fonction principale (Meyer, Nüsslein, 2014). Toujours en contexte de moyenne montagne, cette fois sur les hauts plateaux bourguignons, on peut citer le village de Blessey-Salmais (Côte-d'Or), qui associe, entre le milieu du i ${ }^{\text {er }}$ siècle et la fin du III $^{\mathrm{e}}$ siècle, une quarantaine de petites fermes en enclos mitoyens (Mangin et al., 20oo). On peut y ajouter le site de Waldmatte (Brig-Glis, Valais, Suisse), qui s'étend, en bas de versant des Alpes centrales, sur 1 ha aménagé en terrasses et qui s'inscrit dans la continuité d'une agglomération créée au premier âge du Fer (Paccolat, 1997). Dans les Alpes-Maritimes, le village perché de l'Autreville à Coursegoules, étiré le long d'une ligne de crête, et celui de l'Éouvière à Tourettes-surLoup, sur un sommet et l'un de ses versants, constituent deux autres cas vraisemblables (Bertoncello, Lautier, 2013, p.198-201).

Aux Pays-Bas, chez les Bataves, où les villae sont très peu nombreuses, il existe quelques cas de regroupements de plusieurs fermes (ou maisonsétables), parfois encloses dans un fossé commun - exemple du site de Westerveld à Oss -, ou non - exemple de Beekseweg à Lieshout-, mais pour la qualification desquels on peut hésiter entre hameau et village (Roymans, Heeren, 2004, p. 25-27). Il en est de même en Hollande avec le site de Rijswick, qui associe plusieurs fermes dans un enclos quadrangulaire (Bloemers, 1978), ou encore en Flandre orientale (Belgique) avec le possible village d'Evergem-Luizondoc caractérisé par le rassemblement, le long d'un chemin, de dix-sept fermes sur 16 ha (Bayard, de Clercq, 2013, p.173-175).

À moins qu'il ne s'agisse là, pour reprendre une expression appliquée à la Narbonnaise par Claude Raynaud, de «fermes agglomérées » (2009, p.158). Ce concept ne nous simplifie pas la tâche, mais est bien représentatif des questions posées par la grande diversité des formes de l'habitat rural (Leveau et al., 2009). Des indécisions sont ainsi également perceptibles dans les cas, révélés par l'archéologie préventive,

du village de Longueil-Sainte-Marie (Oise), qui concentre sur 10 ha plusieurs dizaines de constructions à vocation agropastorale (Maréchal, 2009), ou bien du site de l'Épine à Chassieu-Genas (Rhône), toutefois moins étendu mais qui associe deux pôles d'habitat (Coquidé et al., 2009, p. 184-185).

Pour l'Antiquité tardive, et en prétendant encore moins à l'exhaustivité que pour le HautEmpire, les occurrences se font plus rares. On peut signaler l'habitat germanique de SaintOuen-du-Breil, daté du IV ${ }^{\mathrm{e}}$ siècle et à vocation très vraisemblablement agropastorale (Gonzalez et al., 2001), auquel font notamment 
écho d'autres exemples des IV ${ }^{\mathrm{e}}-\mathrm{VI}^{\mathrm{e}}$ siècles en Belgique et aux Pays-Bas (Vermeulen, 2001, p. 57-63). Les recherches récentes ont par ailleurs renouvelé le dossier des établissements - ou villages - perchés de l'Antiquité tardive, dont le panel apparaît cependant assez diversifié, certains abritant des communautés paysannes mais d'autres ayant des fonctions plus développées (mise en défense, rôle dans la christianisation, activités diversifiées...). On en connaît notamment dans le midi de la France (Schneider, 2001; 2007), en Auvergne (Martinez, 2012), dans le Jura (Gandel, 2007; Gandel, Billoin, 2011) ou encore en Gaule du Nord (Brulet, 2008).

\section{Une problématique à développer?}

Ainsi abordé, le village - ou agglomération rurale -, si on s'entend sur la définition qui en a été donnée, paraît bien constituer une forme d'habitat groupé rare et somme toute marginale à l'échelle des Gaules romaines. Certes, il faut pondérer cette impression car, comme on l'a dit, les activités agropastorales peuvent ne laisser que des traces ténues, impossibles à identifier sans recourir à la chimie des sols ou à l'étude fine des macrorestes végétaux et animaux. En contrepoint, il faut toutefois admettre que les agglomérations les mieux connues apparaissent certes étroitement liées au monde rural et même indissociables de celui-ci, mais intègrent, avec parfois des spécialisations, des activités multiformes, artisanales, commerciales, résidentielles, voire religieuses ou administratives. L'exploitation des ressources agricoles y est donc, jusqu'à preuve du contraire, peu présente, comme peuvent aussi en témoigner les concentrations de fermes et/ou de villae régulièrement identifiées dans leur environnement proche et qui laissent peu de place à des terres directement exploitables (voir aussi dans ce sens Ferdière, 2005b, paragr. 6o). Dans cette perspective, qui n'est pas neuve, l'essentiel de l'économie agropastorale serait donc bien principalement du ressort des villae et des fermes. Pour autant, il est très probable que bon nombre des agglomérations abritaient «aussi des populations mettant en valeur directement la terre ou qui participaient à cette exploitation en apportant leur bras aux autres producteurs agricoles » (Ouzoulias, 2006, p. 250). De même, la documentation disponible fait état, de place en place, de l'existence de quartiers spécialisés dans la transformation des produits de la terre, qui viennent toutefois en complément d'autres activités.

Pour aller de l'avant, sans doute est-il temps désormais d'affronter pleinement le sujet en établissant un inventaire des indices susceptibles de plaider - ou non - en faveur d'une implication des habitants des agglomérations dans la production rurale, à la manière de ce qu'a tenté Alain Ferdière en recensant les découvertes d'outils et d'objets à vocation agricole (Ferdière, 2009). Cette problématique est susceptible d'intéresser tout autant les archéologues de la ville, souvent enclins à mettre davantage l'accent sur les traits urbains, que ceux du monde rural, qui se sont surtout concentrés sur les formes de l'habitat dispersé. Mais elle ne pourra être développée efficacement qu'à la condition de l'insérer dans un système de production et de peuplement et en prenant en compte les multiples interactions perceptibles entre les réseaux locaux de l'habitat rural et l'armature urbaine; le village s'inscrivant très exactement à l'interface entre ces deux composantes de l'espace.

\section{Références bibliographiques}

BARET F., 2013, «Les agglomérations antiques du Massif central», in TRÉMENT F. (DIR.), Les Arvernes et leurs voisins du Massif central à lépoque romaine, Clermont-Ferrand, Alliance universitaire d'Auvergne (coll. Revue d'Auvergne, tome 127, $\mathrm{n}^{\circ}$ 606-607), p. 31-71.

Bayard D., De Clerck W., 2013, «Organisation du peuplement et habitats en Gaule du Nord, confrontation de deux exemples régionaux, la Picardie et la Flandre septentrionale », in Fiches J.-L. et al. (DIR.), 2013, p. 161-179.

Bermond I., Buffat L., Fiches J.-L., Garmy P., Pellecuer C., Pomarèdes H., Raynaud C., 2013 « Nîmes en Narbonnaise, essai sur la géographie des territoires à l'échelle de la cité », in FICHES J.-L. et al. (DIR.), 2013, p. 83-98.

Bertoncello F., LAUtier L., 2013, «Formes et organisation de l'habitat en Narbonnaise orientale et dans les Alpes-Maritimes (cités de Fréjus, Antibes, Vence et Briançonnet) », in Fiches J.-L. et al. (DIR.), 2013, p. 195-212.

BLIN O., 2007, «L'agglomération antique de JouarsPontchartrain (Yvelines), Diodurum. Évolution d'un vicus de la cité carnute», in HANoune R. (DIR.), Les Villes romaines du nord de la Gaule, Lille, Revue du Nord (coll. "Art et Archéologie», 10), p. 187-203.
Bloemers J.H.F., 1978, Rijswijk (ZH), "De Bult”. Eine Siedlung der Cananefaten, Den Haag, Staatsuitgeverij (coll. «Nederlandse Oudheden», VIII), 2 vol., $510 \mathrm{p}$

Bouet A., TASSAUX F., 2005, «Les agglomérations secondaires d'Aquitaine à l'époque julioclaudienne", in L'Aquitaine et l'Hispanie septentrionale à l'époque julio-claudienne. Organisation et exploitation des espaces provinciaux, Bordeaux, Aquitania (coll. Suppl à Aquitania, 13), p. 267-299.

BRULET R., 2008, «Fortifications de hauteur et habitat perché de l'Antiquité tardive au début du haut Moyen Âge, entre Fagne et Eifel», in STEUer H., Bierbrauer V., Hoeper M. (eds.),

Höhensiedlungen zwischen Antike und Mittelalter von den Ardennen bis zur Adria, Berlin/New York, Walter de Gruyter (coll. «Ergänzungsbände zum Reallexikon der Germanischen Altertumskunde», 58), p. 13-70

Brun J.-P., BorréANi M., 1999, Le Var, Paris, MSH (coll. «Carte archéologique de la Gaule», 83/2), 984 p.

Brunet R., Ferras R., Rhéry H., 1993, Les Mots de la géographie. Dictionnaire critique, Montpellier, Reclus/La Documentation française, $517 \mathrm{p}$.

Chevallier R. (ÉD.), 1976, Le Vicus gallo-romain, Actes du colloque de Tours, novembre 1976, Tours,
Presses universitaires de Limoges (coll. «Caesarodunum», 11), 334 $\mathrm{p}$

Coquidé C., Frascone D., Ramponi C., ThollonPommerol C., 2009, « Habitats ruraux antiques à interpréter au nord de la Narbonnaise ", in LEVEAU P. et al., 2009, p.179-198.

Cribellier C., 2013, "Le réseau des agglomérations antiques dans les cités du centre de la Gaule», in LORANS É., Rodier X. (DIR.), 2013, p. 433-450.

Cribellier C., Ferdière A. (Dir.), 2012, Agglomérations secondaires antiques en région Centre. Actes de la table ronde d'Orléans, Tours, FERACF (coll. Suppl. à la Revue archéologique du centre de la France, 42), $187 \mathrm{p}$.

DONDIN-PAYRE M., 2012, «L'organisation du territoire civique en Gaule, l'exemple des cités des Gaules centrales », in Cribellier C., Ferdière A. (Dir.), 2012, p. 153-163.

FAVORY F., 2012, « La dure condition des agglomérations secondaires ", Les Nouvelles de l'archéologie, 127, p. 40-44.

Ferdière A., 2005a, Les Gaules. II e s. av. J.-C. $-V^{e} s$. ap. J.-C., Paris, A. Colin (coll. U), 447 p.

Ferdière A., 2005b, «Chronique "À travers les campagnes de la Gaule romaine", XII », Revue archéologique du centre de la France, 44, [en ligne] <http://racf.revues.org/543>.

Ferdière A., avec la collaboration de BENDER H. et 
Lemaire P., 2009 : «Recherche sur les contextes de découverte d'outillage agricole et objets liés au travail et à la production rurale en Gaule romaine», in LeVEau P. et al., 2009, p. 81-107.

FERDIÈrE A., 2013, « 20 ans après... ou les mécomptes de Barcelone. Bilan de 20 ans de recherches sur la Gaule rurale», [en ligne] <http://ager.hypothèses. org $>$.

Ferdière A., Gandini C., Nouvel P., Collart J.-L., 2010, «Les grandes villae "à pavillons multiples alignés" dans les provinces des Gaules et des Germanies : répartition, origine et fonctions », Revue archéologique de l'Est, 59, p. 357-446.

Février P.-A., Fixot M., Goudineau C., Kruta V., 1980, La ville antique des origines au IX $X^{e}$ siècle, in Duby G. (DIR.), Histoire de la France urbaine, t. I, Paris, Seuil (coll. «l'Univers historique) », 605 p.

Fiches J.-L., 2013, «Mosaïque de paysages et diversité des territoires», in FICHES J.-L. et al. (DIR.), 2013, p. 367-375.

FICHES J.-L. (DIR.), 2002, Les Agglomérations galloromaines du Languedoc-Roussillon, t. I et II, Lattes, ADAL (coll. «Monographies d'archéologie méditerranéenne », 13 et 14), $994 \mathrm{p}$.

Fiches J.-L., Plana-Mallart R., Revilla Calvo V. (DIR.), 2013, Paysages ruraux et territoires dans les cités de l'Occident romain. Gallia et Hispania, Montpellier, Presses universitaires de la Méditerranée (coll. «Mondes Anciens »), 396 p.

FichtL S., 2005, La ville celtique. Les oppida de 150 av. J.-C. ̀̀ 15 ap. J.-C., Paris, Errance, 238 p.

Fichtl S., 2013, «Les agglomérations gauloises de la fin de lâge du Fer en Europe celtique (III $-I^{\mathrm{er}}$ siècle av. J.-C.) », in GARCIA D. (DIR.), L'Habitat en Europe celtique et en Méditerranée préclassique: domaines urbains, Paris, Errance, p. 19-44.

GANDEL P., 2007, «Recherches sur les sites de hauteur de l'Antiquité tardive dans le département du Jura », in Belet-Gondat C., Mazimann J.-P., Richard A., SChifferdeCKER F. (DIR.), Mandeure, sa campagne et ses relations d'Avenches à Luxeuil et d'Augst à Besançon, Besançon/ Porrentruy, Presses universitaires de FrancheComté (coll. «Annales littéraires de l'université de Besançon», 816 - série Environnement, sociétés et archéologie, 10; Cahier d'archéologie jurassienne, 2o), p. 245-252.

GANDEL P., Billoin D., 2011, «Le site de "Gaillardon" à Ménétru-le-Vignoble (Jura): un établissement de hauteur de l'Antiquité tardive et du haut Moyen Âge », Revue archéologique de l'Est, 6o, p. 313-421.

Garcia D., 2004, La Celtique méditerranéenne. Habitats et sociétés en Languedoc et Provence du VIII ${ }^{e}$ au $I^{e}$ S. av. J.-C., Paris, Errance, 207 p.

GARMY P., 2012a, «Les mots et la chose: à propos des agglomérations secondaires antiques », in Cribellier C., Ferdière A. (Dir.), 2012, p. 183-187.

GARMY P., 2002, «Villa-vicus: une question d'espace», in GARMy P., LeVeau P. (DIR.), 2002, p. 27-37.

GARMY P., 2012b, Villes, réseaux et systèmes de villes. Contribution de l'archéologie, Paris, Errance, $329 \mathrm{p}$

GARMY P., KADDOURI L., 2013, «Modélisation spatiale d'un réseau urbain antique: à propos des villes de la cité de Nîmes », in Lorans É., Rodier X. (DIR.), 2013, p. 479-493.

Garmy P., LeVEAu P. (DIR.), 2002, «Dossier villa et vicus en Gaule Narbonnaise », Revue archéologique de Narbonnaise, 35, p. 1-317.

Genin M., LAVENDHOMme M.-O., 1997, Rodumna (Roanne, Loire), le village gallo-romain. Évolution des mobiliers domestiques, Paris, MSH (coll. «Documents d'archéologie française», 66), 289 p.

Gonzalez V., Ouzoulias P., Van Ossel P., 2001, «Saint-Ouen-du-Breuil (Haute-Normandie, Frankreich). Eine germanische Siedlung aus der Mitte des 4. Jahrhunderts in der Lugdunensis Secunda», Germania, 79, p. 43-61.

HeRvé C., 2012, «Les termes vicus, castrum, castellum dans l'œuvre de Grégoire de Tours chez les Turons, les Carnutes et les Bituriges ", in Cribellier C., Ferdière A. (DIr.), 2012, p. 145-151.

LEVEAU P., 1993, «Agglomérations secondaires et territoires en Gaule Narbonnaise », Revue archéologique de Narbonnaise, 26, p. 277-299.

LEVEAU P., 2002, «Introduction: les incertitudes du terme villa et la question du vicus en Gaule
Narbonnaise », in Garmy P., LeVeau P. (DIR.), 2002, p. 5-26.

LEVEAU P., 2005-2006, «Les agglomérations de la cité de Vienne, un dossier en devenir ", in LeVEAu P., RÉMY B. (DIR.), 2005-2006, p. 157-169.

LeVEAU P., 2012: «Vicus, "agglomération secondaire". Des mots différents pour une même entité? », in Cribellier C., Ferdière A. (DIR.), 2012, p. 165-175.

LEVEAU P., 2013, «Du bon usage de l'anachronisme dans la recherche archéologique sur les agglomérations protohistoriques et gallo-romaines ", in GARCIA D. (DIR.), L'Habitat en Europe celtique et en Méditerranée préclassique: domaines urbains, Arles/Paris, Errance, p. 9-18.

LeVEAu P., RÉMY B. (ÉD.), 2005-2006, «Dossier: Les agglomérations urbaines de la cité antique de Vienne", Revue archéologique de Narbonnaise, 38-39, p. 6-169.

Leveau P., Raynaud C., Sablayrolles R., TRÉMENT F., 2009, Les Formes de l'habitat rural gallo-romain. Terminologies et typologies à lépreuve des réalités archéologiques, Actes $d u$ colloque AGER VIII, Toulouse 2007, Bordeaux, Aquitania (coll Suppl. à Aquitania, 17), 396 p.

Lorans É., Rodier X. (DIR.), 2013, Archéologie de lespace urbain, Tours/Paris, Presses universitaires François-Rabelais/CTHS (coll. «Perspectives Villes et Territoires »), $535 \mathrm{p}$

MANGIN M., JACQUET B., JACOB J.-P. (DIR.), 1986, Les Agglomérations secondaires en Franche-Comté romaine, Paris, Les Belles Lettres (coll. «Annales littéraires de l'université de Besançon », 337), 261 p.

MANGIN M., TASSAUX F., 1992, «Les agglomérations secondaires de l'Aquitaine romaine», in Villes et agglomérations urbaines antiques du sud-ouest de la Gaule. Histoire et archéologie. Deuxième colloque Aquitania, Bordeaux, 199o, Bordeaux, Aquitania (coll Suppl. à Aquitania, 6), p. 461-496, 502-511.

Mangin M., Courtadon J.-L., Fluzon P., DE LACOLS É. , 200o, Village, forges et parcellaire aux sources de la Seine. Lagglomération antique de Blessey-Salmaise (Côte-d'Or), Besançon, Presses universitaires franc-comtoises (coll. «Annales littéraires de l'université de Franche-Comté», vol. 6999 - série Environnement, sociétés, archéologie, 2), $518 \mathrm{p}$.

MARÉCHAL D., 2009, «Le village de Longueuil-SainteMarie et les autres formes d'implantation agricole dans la moyenne vallée de l'Oise », in LeveAu P. et al., 2009, p. 199-215

MARTinez D., avec la collaboration de CHABERT S. 2012, «Le site de La Couronne à Molles (Allier): un établissement de hauteur de l'Antiquité tardive et du haut Moyen Âge. Bilan de la deuxième campagne de recherches ", Bulletin du centre d'études médiévales, 16, [en ligne] <http://cem. revues.org/12382>.

MASSY J.-L. (DIR.), 1997, Les Agglomérations secondaires de la Lorraine romaine, Paris, Les Belles Lettres (coll. «Annales littéraires de l'université de Franche-Comté», 647), 433 p.

Meyer N., Nüsslein A., 2014, «Une partie de la campagne gallo-romaine du Haut-Empire des cités des Médiomatriques et des Triboques préservée par la forêt: les habitats et parcellaires des Vosges du nord (Moselle et Bas-Rhin) de part et d'autre du seuil de Saverne », [en ligne] <http://rurland. hypotheses.org.>, $37 \mathrm{p}$

MonTeil M., 2012, Contribution à l'étude des agglomérations secondaires des Gaules romaines. Les cités de l'ouest de la province de Lyonnaise (Bretagne et Pays de la Loire), Mémoire $\mathrm{d}$ 'habilitation à diriger des recherches, Histoire, Université François-Rabelais, Tours, 363 p.

Ouzoulias P., 2010, «Les campagnes gallo-romaines: quelle place pour la villa?», in Ouzoulias P. Tranoy L. (DIR.), Comment les Gaules devinrent romaines, Paris, La Découverte/Inrap, p. 189-211.

Ouzoulias P., Pellecuer C., Raynaud C., Van Ossel P., GARMY P. (DIR.), 2001, Les Campagnes de la Gaule à la fin de l'Antiquité, Actes du colloque AGER IV, Montpellier, 11-14 mars 1998, Antibes, APDCA, 640 p

PaCcolat O., 1997, «Le village gallo-romain de BrigGlis/Waldmatte», Archéologie suisse, 20, p. 25-36.
Petit J.-P., Brunella P., 2005, Bliesbruck-Reinheim. Celtes et Gallo-Romains en Moselle et en Sarre, Paris, Errance (coll. Hauts lieux de l'Histoire), $223 \mathrm{p}$

Petit J.-P., Mangin M. (DIR.), avec la collaboration de BRUNELLA P., 1994, Les agglomérations secondaires. La Gaule Belgique, les Germanies et l'Occident romain. Actes du colloque de Bliesbruck-Reinheim/ Bitche 1992, Paris, Errance (coll. «Archéologie aujourd'hui»), 294 p.

PÉTRY F., 1997, «Les agglomérations des sommets vosgiens », in MASSY J.-L. (DIR.), 1997, p. 399-405.

Pomarèdes H., Compan M., Ginouvez O., Jung C., 2008, «Viticulture et équipements de production dans la cité de Lodève durant le Haut-Empire», Revue archéologique de Narbonnaise, 41, 2008, p. 7-41.

Py M., 2012, Les Gaulois du Midi. De la fin de lâge du Bronze à la conquête romaine, Paris, Errance, $400 \mathrm{p}$.

RAYNAUD C., 2002, «De la conquête romaine au Moyen Âge», in FiCHES J.-L. (DIR.), 2002, p.39-53.

RAYNAUD C., 2003 « Le système de peuplement de l'âge du Fer au Moyen Âge ", in Bats M., Dedet B., Garmy P., Janin T., Raynaud C., Schwaller M. (DIR.), Peuples et territoires en Gaule méditerranéenne. Hommage à Guy Barruol, Montpellier, Université Paul-Valéry (coll. Suppl. à la $R A N, 35)$, p. 323-354.

RAYNAUD C. (DIR.), 2002, Le Village gallo-romain et médiéval de Lunel-Viel. Les fouilles du quartier ouest, Paris, Les Belles Lettres (coll. «Annales littéraires de l'université de Besançon », 422), 353 p

RAYNAUd C. (DIR.), 2007, Archéologie d'un village languedocien. Lunel-Viel (Hérault) du $\mathrm{I}^{\mathrm{er}}$ au $\mathrm{XVIII}^{\mathrm{e}}$ S., Lattes, ADAL (coll. «Monographies d'archéologie méditerranéenne», 22), 407 p.

Raynaud C., Pomarèdes H., Manniez Y., 2009, «Fermes gallo-romaines de la province de Narbonnaise ", in LeVEAU P. et al., 2009, p.141-165.

Roymans N., HeEREN S., 2004, «L'archéologie des habitats ruraux romains aux Pays-Bas. Bilan de cent années de recherches », Revue du Nord Archéologie de la Picardie et du nord de la France, 86, 2004, p. 19-36.

SCHNEIDER L., 2001, «Oppida et castra tardo-antiques. À propos des établissements de hauteur de la Gaule méditerranéenne», in Ouzoulias et al. (DIR.), 2001, p. 433-448.

SCHNEIDER L., 2007, « Rythmes de l'occupation rurale et formes de l'habitat dans le sud-est de la France entre Antiquité et Moyen Âge (IV ${ }^{\mathrm{e}}$-VIII ${ }^{\mathrm{e}} \mathrm{s}$.) : essai de synthèse», Gallia, 64, p.11-56.

TARPIN M., 1999 : «Oppida ui capta, uici incensi. Les mots latins de la ville», Latomus, 52, p. 279-297.

TARPIN M., 2002 : Vici et pagi dans l'Occident romain, Rome, École française de Rome (coll. «École française de Rome», 299), 470 p.

TARPIN M., 2009, «Organisation politique et administrative des cités d'Europe occidentale sous l'Empire», in Rome et l'Occident. II siècle avant J.-C. - II siècle après J.-C., Toulouse, Presses universitaires du Mirail (coll. «Pallas», 80), p. 127-14.6.

TARPIN M., 2012, «Vici et agglomérations secondaires quelques faux problèmes ", in CRIBELLIER C., FERDIÈRE A. (DIR.), 2012, p. 177-182.

Vermeulen F., 2001, «Les campagnes de la Belgique septentrionale et des Pays-Bas méridionaux aux IV et $\mathrm{V}^{\mathrm{e}}$ siècles ", in Ouzoulias et al. (DIR.), 2001, p. 45-68.

VAN OSSEL P., 2010, «De la "villa" au village: les prémices d'une mutation », in YANTE J.-M. Bultot-Verleysen A.-M. (Dir.), Autour du «village». Établissements humains, finages et communautés rurales entre Seine et Rhin (IV $V^{e}$ $X I I I^{e}$ siècles). Actes du colloque international de Louvain-la-Neuve, 16-17 mai 2003, Louvain-laNeuve, Institut d'études médiévales de l'université catholique de Louvain (coll. «Publications de l'Institut d'études médiévales, 25), p. 219-236.

Vidal De la Blache P., 1922, Principes de géographie humaine publiés d'après les manuscrits de l'auteur par E. de Maronne, Paris, A. Colin, 1922, 327 p. 